\title{
Jogos Digitais e a Dependência Química: Um Mapeamento Sistemático da Literatura
}

\author{
André Nunes $^{1}$ Helder Cognaco de Oliveira ${ }^{1}$
}

Maria de Lourdes de Souza ${ }^{2}$ Marcelo da Silva Hounsell ${ }^{1}$

\author{
${ }^{1}$ Laboratory for Research on Visual Applications (LARVA) - Departamento de \\ Ciência da Computação (DCC) - Universidade do Estado de Santa Catarina (UDESC), \\ Joinville, SC - Brasil
}

${ }^{2}$ Programa Pós Graduação em Enfermagem, Universidade Federal de Santa Catarina (UFSC), Instituto Repensul, Florianópolis, Brasil. \{dekonunesss,heldercdo\}@gmail.com, marcelo.hounsell@udesc.br,
repensul@uol.com.br

\begin{abstract}
Drug addiction has become a health issue everywhere. People from all ages and social classes are subject to this problem. This paper aims to identify scientific production about educational games in the context of drug addiction through a systematic literature mapping. The search was done in the area of Computer Science through the following sources: IEEE Xplore, Web of Science, ACM Digital Library and Science Direct. The result was 957 published papers. After applying mapping criteria, 7 papers were selected. Special care was taken to differentiate among interactive graphical applications those that were in fact games. Among games found, 5 were digital, of academic origin and no preferred genre. Only 2 were made in Brazil and are in Portuguese. The majority focus on children and teens and prevention of drugs in general. The scenario analyzed shows that there is an opportunity for more digital games in the context of drug addiction.
\end{abstract}

Keywords: Digital Games, Drugs, Drug Addiction, Therapeutic Communities.

Resumo. O abuso de drogas se constitui em um problema de saúde pública no mundo inteiro. Incide em pessoas de todas as idades e de todas as classes sociais. Este artigo objetiva identificar a produção científica sobre jogos educacionais no contexto da dependência química através de um mapeamento sistemático de literatura. A busca foi realizada na área da Ciência da Computação com as seguintes fontes de consulta: IEEE Xplore, Web of Science, ACM Digital Library e ScienceDirect. Foram encontrados 957 textos publicados. Após aplicados os critérios de mapeamento foram selecionados 7 artigos. Cuidado especial foi tomado para diferenciar entre aplicações gráficas interativas aquelas que eram realmente jogo. Dentre os jogos encontrados 5 eram digitais, sem um gênero preferido, todos de origem académica. Apenas 2 deles foram feitos no Brasil e são em português. A maioria trata prevenção para crianças e adolescentes e drogas em geral. $O$ cenário analisado demonstra que existe uma oportunidade para mais Jogos Digitais no contexto da dependência química. 
CBIE-LACLO 2015

Anais do XXVI Simpósio Brasileiro de Informática na Educação (SBIE 2015)

Palavras-Chave: Jogos Digitais, Drogas, Dependência Química, Comunidades Terapêuticas.

\section{Introdução}

Os transtornos por uso de substância, também denominados de dependência de drogas são dependência química, se constituem em doenças de ordem biológica, psicológica e social com comprometimento ao indivíduo, família e sociedade. A auto administração de drogas pode estar associada ou não ao conhecimento sobre seus prejuízos.

Existem vários programas de reabilitação e se diferenciam em seus objetivos, metodologias e ideologias [IMESC, 2014]. Os programas de reabilitação precisam ser diferentes por causa do indivíduo, da cultura, condição social e financeira que o Dependente Químico (DQ) se encontra; todos esses aspectos têm que ser levados em conta para se ter o tipo certo de programa terapêutico.

O problema com abuso de drogas acaba criando um indivíduo sem motivação, interfere em sua cognição, deixa seu humor debilitado, aumenta os riscos de problemas de saúde e pode trazer a morte. O estilo de vida de um DQ prejudica seus estudos, causa problemas mentais e, às vezes, leva ao mundo do crime [Hawkins, 1992].

Os adolescentes são particularmente vulneráveis pois estão em fase de desenvolvimento e em sua maioria são suscetíveis a pressões de seus colegas e não sabem dizer não. A iniciação precoce no uso de drogas é considerada um fator de risco no desenvolvimento de disfunções de uso de substâncias, comorbidade, criminalidade e comprometimento do desempenho escolar [Rodriguez, Teesson e Newton, 2013]. Portanto, o efeito das drogas em adolescentes é amplo e justifica uma ação preventiva o mais cedo possível.

Jogos digitais são de grande interesse para jovens, devido a sua natureza prazerosa, interativa e visualmente atraente. A atual geração de jovens pode ser caracterizada como Nativos Digitais [Prensky, 2001] o que significa que estes jovens cresceram cercados de todos os tipos de acessórios digitais como computadores, smartphones, tablets e, jogos digitais. Assim sendo, estes compoêm uma mídia natural e comum para eles quando precisam lidar com o mundo real.

Então surge a questão de identificar se, como e quantos são os jogos educacionais (principalmente os digitais) que estão sendo usados no contexto do uso de substâncias psicoativas, tanto na prevenção do uso primário, com os jovens quanto no acompanhamento de tratamentos ou até prevenção de recaída.

$\mathrm{Na}$ seção 2 serão apresentados conceitos sobre o que caracteriza um jogo. $\mathrm{Na}$ seção 3 detalhasse as justificativas deste estudo, e na seção 4 o mapeamento, explicando o protocolo de pesquisa utilizado e como foram aplicados os seus passos. Na seção 5 são apresentadas discussões e na seção 6 as conclusões. No Apêndice 1 são apresentados os artigos encontrados.

\section{Caracterizando um "jogo"}

A diferenciação entre um software de Realidade Virtual, Realidade Aumentada, Simulação ou outro software interativo e um jogo digital nem sempre é trivial. Para 
realizar esta diferenciação, foram utilizados os conceitos de [Prensky, 2001], onde um jogo basicamente consiste de:

(i) Regras, que diferenciam um jogo de uma simples brincadeira livre, pois um jogo é uma atividade organizada, com certas delimitações impostas;

(ii) Objetivos e metas, que geralmente são descritos no começo do jogo, motivam um jogador a se engajar na atividade e especificam o que se espera dos jogadores. Mesmo que não se tenham metas, que seja algo para se brincar mas em várias formas distintas dependentes de seu próprio objetivo, então este item está atendido;

(iii) Resultados e feedback, são fundamentais em jogos digitais, pois é através destes que se mede o progresso de um jogador acerca dos objetivos do jogo. O feedback possibilita que o jogador saiba imediatamente se suas ações geraram um resultado positivo ou negativo e, portanto, estão de acordo com as regras do jogo. Normalmente este item se apresenta na forma de um placar, outra forma de pontuação ou, até formas mais sutis como, por exemplo, um indicador "quente-frio" a medida que o jogador está perto-longe do objetivo. Além disso, o feedback possibilita que o jogador compare o seu desempenho com outros jogadores. Segundo Prensky (2001), é através das percepções obtidas pelo feedback que ocorre o processo de aprendizagem em um jogo;

(iv) Conflito/desafio, que é a definição do quê se deve fazer para alcançar os objetivos do jogo, respeitando as regras impostas. Representam os problemas que se está tentando resolver. Esta característica pode ser demonstrada de várias formas, como competição entre jogadores, níveis de dificuldade, entre outros;

(v) Interação, que é a característica de um jogo que pode ser observada sob duas óticas diferentes. Primeiramente, existe a interação do jogador com o computador, que é tratada através do feedback; A segunda porém, é o aspecto inerente à socialização através dos jogos;

(vi) Por fim, a representação ou enredo sobre do que o jogo se trata. Nesta característica, inclui-se qualquer narrativa ou elementos de enredo do jogo. Alguns teóricos julgam que a representação é a essência do que constitui um jogo, enquanto outros acham que é apenas uma ferramenta auxiliar do jogo.

Os três primeiros itens - ou de modo simplista as regras, o objetivo e as situações de vencer ou perder -formam a clássica e bem-aceita definição de um jogo, que existe há séculos, conforme destaca Prensky (2001).

\section{Justificativas}

Segundo o relatório da ONU (Organização das Nações Unidas, 2005), o Brasil é o segundo maior mercado de drogas das Américas, com mais de 800 mil usuários, apenas atrás dos Estados Unidos que possuem seis milhões de consumidores. Porém em Junho 2012 a ONU afirmou que o número de usuários, tanto de crack quanto de cocaína, aumentaram muito nas terras brasileiras.

Muito se tem feito no Brasil para que as pessoas se previnam contra o uso dessas substâncias ilegais, ou até mesmo as legais, como álcool: desde a prevenção em escolas com o uso de programas como o PROERD (Programa Educacional de Resistência às 
Drogas e à Violência), até conselhos em casa perante a família. Porém são drogas como crack, (cujo seu efeito de dependência é mais rápido que outras substancias ilícitas) que acabam dificultando a prevenção por serem fáceis de serem produzidas, encontradas e comercializadas.

Após o indivíduo ter contato com estes tipos de substâncias, o mesmo pode começar a ter problemas em vários aspectos do seu cotidiano, como o próprio convívio na sociedade e com sua família, destruindo sua vida de uma forma lenta e dolorosa. Visando recolocar cidadãos que desenvolveram dependência às drogas na sociedade, surgiram as Comunidades Terapêuticas (CTs) que, em sua maioria, são instituições privadas, sem fins lucrativos e ajudadas pelo poder público na maioria dos casos, oferecendo assim o acolhimento para essas pessoas que tiveram problemas decorrente ao uso de substâncias. Dentro das CTs os profissionais aplicam um programa terapêutico aos dependentes de substancias psicoativas durante um período estabelecido de acordo com a organização de cada instituição.

Vê-se potencial de uso de Jogos Digitais (JD) tanto em processos preventivos quanto terapêuticos no combate à drogadição. A psicologia associada ao uso de JD foi explorada em [Boyle, Connolly e Hainey, 2011] quanto a sua capacidade de promover mudanças de atitude e comportamento considerando que os psicólogos demonstram ser receptivos à ideia de usar jogos no contexto do ensino. Estes profissionais consideram que o potencial do uso de JD no ensino decorre do otimismo de que as características motivacionais dos jogos de entretenimento possam ser usadas. Não obstante ao aspecto motivacional, os JD oferecem oportunidades e atividades que são consistentes com teorias modernas de aprendizagem efetiva [op. cit., pag 72]: aprendizagem ativa e vivencial, situacional e orientada a solução de problemas, objetivos claros com feedback imediato e a construtivista.

Recentemente, Rodriguez, Teesson e Newton (2013) buscaram artigos envolvendo jogos no contexto da prevenção. Apesar da busca parecer ampla, o trabalho focava exclusivamente em jogos que haviam sido testados com adolescentes. Isto resultou em apenas 6 jogos "universais" (independente dos fatores de risco envolvidos) com ênfases educacionais e cuja avaliação foi de conhecimento. Cinco destes jogos demonstraram aumento quantitativo de conhecimento adquirido com o uso do jogo. Nada muito detalhado sobre aspectos dos jogos em si foi relatado o que dificulta saber que tipo de jogo tem sido usado e, com foco em adolescentes e prevenção, limita a análise do papel dos jogos como um todo no contexto das drogas.

No artigo de Rodriguez, Teesson e Newton (2014) os mesmos relatam que a cada ano que se passa a comunidade cientifica tende a aceitar e afirmar que os jogos não tem apenas o objetivo de entretenimento mas, com o uso adequado dos mesmos, é possível notar a importância dos jogos no âmbito de ensinar e ao mesmo tempo se divertir jogando. Mesmo com a pouca quantidade de jogos encontrados, ainda assim é possível afirmar que os jogos já existentes ajudam em, ao menos, aumentar o conhecimento dos jogadores.

A comunidade científica reconhece a importância e o crescimento de JD e que já existem iniciativas do uso de jogos sendo aplicados no contexto da Dependência Química (DQ). Entretanto, os trabalhos anteriores não trataram recentemente, de maneira abrangente, do uso JD contra o uso de drogas. 


\section{Mapeando Jogos para a Dependência Química}

Um Mapeamento Sistemático de Literatura (MSL) visa efetuar uma pesquisa científica em largura, não em profundidade ([Kitchenham e Charters, 2007], [Petersen et al., 2008]). A vantagem em seguir um protocolo de MSL consiste na possibilidade de que outros pesquisadores podem reproduzir os passos do mapeamento e chegarem aos mesmos resultados, o que agrega rigor científico na pesquisa.

No presente mapeamento além das palavras-chave, filtros e objetivos foram definidos para serem utilizados nos Mecanismos de Busca Acadêmico (MBA). Os filtros utilizados foram:

- Material em inglês ou português;

- Publicações de periódicos e conferências;

- Artigos completos;

- Artigos de até 10 anos de idade.

Foi determinado o período de 10 anos uma vez que a tecnologia (e até o visual associado) na área de JD vem evoluindo muito, de tal forma que um JD de mais de uma década de idade é considerado velho e ultrapassado só por causa de seu visual.

\subsection{Jogos no Contexto das Drogas}

O objetivo principal deste mapeamento é "identificar jogos educacionais relacionados ao combate ao uso de drogas". Através dos dados que serão levantados acerca destes jogos, pretende-se atender os seguintes objetivos secundários:

- Como são esses jogos (sua origem, seu gênero). Como se apresentam estes jogos (sua mídia, licença de uso)?

- Como se inserem estes jogos no contexto dos dependentes químicos (público alvo, drogas envolvidas, fase da dependência química)?

- Qual a efetividade desses jogos (métodos de avaliação e resultados)?

Para definir a busca efetivamente realizada nos MBAs, foram criadas palavraschave abrangentes tendo em vista a aplicabilidade do tema de DQ em diversas áreas de estudo como medicina, computação e biologia. A frase de busca utilizada foi definida como:

(game? OR jogo? OR "virtual reality" OR "realidade virtual”) AND ((drug?

OR substance?) NEAR (*use OR addiction)) OR ((droga? OR substância?)

NEAR ((uso) OR vício OR dependência)

A utilização dos termos "realidade virtual" e "virtual reality" deu-se devido a pratica na área da saúde de considerar estes termos sinonimos de jogos digitais.

Os MBA selecionados foram o IEEE Xplore, ACM Digital Library e Web of Science e Science Direct, devido a serem MBAs com uma grande massa de publicações, e serem relevantes e bem conceituados na área de Ciência da Computação, que contempla a área de JD também.

Os critérios de inclusão (CI) e exclusão (CE) tem como função definir quais artigos encontrados através das buscas nos MBAs serão efetivamente mapeados. Dessa forma, os artigos que serão analisados devem atender a, pelo menos um, dos seguintes CIs: 
- CI-1: Artigo sobre um jogo com foco no combate à dependência química a drogas psicoativas;

- CI-2: Artigo deve descrever o JD ou um modelo de jogabilidade;

Em compensação, para que um artigo seja analisado, nenhum dos CEs devem ser identificados nele, conforme:

- CE-1: Artigo duplicado;

- CE-2: Artigo sem acesso ou não é um artigo completo;

- CE-3: Artigo não apresenta a educação como propósito principal.

CIs e CEs foram aplicados com base na leitura do título e abstracts dos artigos encontrados. Quando não foi possível concluir sobre a aplicabilidade de determinado critério através do abstract, então avaliou-se a introdução e conclusão.

\subsection{Resultados do Mapeamento}

Foram obtidos 957 artigos na busca dos 4 MBAs, conforme monstrado na Tabela 1 que mostra o número de artigos selecionados após cada etapa. Todos estes artigos foram lidos e analisados quanto as CIs e CEs.

\begin{tabular}{|l|l|l|l|}
\hline MBA & Resultados & Após CE & Após CI \\
\hline Web of Science & 81 & 78 & 3 \\
\hline IEEE Explore & 129 & 129 & 0 \\
\hline ScienceDirect & 730 & 729 & 1 \\
\hline ACM Digital Library & 9 & 9 & 0 \\
\hline $\begin{array}{l}\text { Buscas não estruturadas } \\
\text { em artigos do tipo } \\
\text { revisão }\end{array}$ & 8 & 5 & 3 \\
\hline TOTAL & 957 & 950 & 7 \\
\hline
\end{tabular}

Tabela 1: Dados da pesquisa

Foram inseridos outros 3 artigos obtidos fora dos MBAs por meio de uma busca complementar em artigos do tipo revisão, como o trabalho de [Boyle, Connolly e Hainey, 2011], de onde percebeu-se que alguns jogos ali identificados e discutidos, atendiam aos critérios deste mapeamento. Após a análise de todos os resultados, foram encontrados 7 artigos.

Durante a fase de busca e seleção, de todos os artigos encontrados na busca em apenas um foi necessária a leitura por completo. A leitura foi feita para analisar melhor a pertinência do trabalho, mas constatou-se que não, não se tratava de um jogo, mas um simulador do tráfico de drogas.

Metade destes artigos são do mesmo projeto criado na universidade de Rice e utilizam a mesma estratégia de ensino. A Tabela 2 lista as informações que foram levantada nos 7 artigos encontrados. $\mathrm{O}$ detalhamento destas informações devidamente identificadas em cada um dos 7 artigos mapeados está mostrado no Apêndice 1. Todos 
os jogos foram criados como projetos em universidades, sendo que destes jogos, 4 $(57,1 \%)$ são online e gratuitos. Os outros $3(42,9 \%)$, são pagos.

Tabela 2: Dados Mnemônicos

\begin{tabular}{|l|l|}
\hline IGJ & Identificar o Gênero de Jogo \\
\hline ADN & Autodenominação \\
\hline ORJ & Origem do Jogo \\
\hline MID & Mídia de distribuição do Jogo \\
\hline LIC & Licença do Jogo \\
\hline DAL & Droga(s) alvo \\
\hline PAL & Publico alvo \\
\hline EDQ & Etapa da dependência onde o jogo foi aplicado \\
\hline MAV & Métrica adotada para Avaliação \\
\hline RES & Resultados alcançados \\
\hline
\end{tabular}

Dentre os jogos encontrados no MSL apenas 2 deles foram feitos no Brasil e são em português. Três jogos (N-SquadWeb Adventure, The Reconstructors: Uncommon Scents, Reconstructor: Nothing to Rave About) fazem parte de uma sequência de 4 jogos contra drogas, mas apenas 3 entraram no mapeamento devido ao fato de que o artigo que apresenta o quarto jogo ser do ano de 2002, não passando pelos filtros do mapeamento. Os tipos de jogos são bem variados, dentre eles um que faz uso de cartas, outro de um tabuleiro e os demais são softwares. Ou seja, dos 7, 5 são JD.

\section{Discussão}

Uma dificuldade durante o MSL foi diferenciar dentre os artigos encontrados, quais realmente apresentavam um jogo, quais apresentavam uma aplicação de simulação, Realidade Virtual ou outro tipo de aplicação gráfica 3D mas não com características de jogo. Para a diferenciação, foram usadas as características vistas na seção 2 .

A maioria dos projetos têm origem acadêmica com uma tendência de criação de jogos para smartphone, também chamados de mHealth. Como se pode depreender do que é apresentado no Apêndice 1 (tabela após as referências), não se pôde identificar uma preferência por algum gênero de jogo em specífico (ver linha IGJ) mas quanto a auto-denominação (ver linha $\mathrm{ADN}$ ), parece ser uma tendência a denominação "educacional". Dos jogos que deixam isto claro, todos se apresentam gratuitamente para uso, o que favorece a disseminação, apesar da língua de origem. Outro aspecto que concorre para a disseminação é o foco (ver linha DAL) contra o uso de drogas em geral e, em segundo lugar, álcool (sendo este um dos mais comuns problemas de DQ).

Desde o início da pesquisa tinha-se a ideia de que seria simples e muito rápido encontrar jogos com tais critérios, mas observou-se que é muito baixo o número de jogos com intuito de ajudar DQs, e ainda obteve-se um resultado nulo para jogos utilizados em CTs. Grande parte dos artigos tende a considerar CTs como lugares onde é possível tratar obesidade, problemas cardíacos, recuperação de pessoas que tiveram derrame, e até mesmo pessoas com AIDS, dificultando assim a ideia de encontrar uma definição de CT correta para a pesquisa. 


\section{Conclusão}

A utilização de jogos, principalmente os digitais, como forma de auxiliar na atenção ao dependente químico (DQ) vai ao encontro das últimas tendências tecnológicas, motivacionais e pedagógicas. Mas, por ser uma iniciativa inovadora, precisa ser avaliada cientificamente quanto a sua utilidade, facilidade e formas de fazer.

Este artigo fez um mapeamento sistemático de literatura em busca de jogos sendo usados no contexto da DQ. Esta busca foi feita em mecanismos de busca acadêmica (MBA) da área da computação, uma vez que buscava-se compreender como o desenvolvimento deste tipo de software tem sido feito. Após a análise dos 957 artigos em 4 MBA e de artigos do tipo revisão, com a restrição de jogos criados nos últimos 10 anos, a busca localizou apenas 7 jogos, onde 5 destes eram em mídia digital.

Mesmo sendo uma quantidade pequena de jogos, algumas análises podem ser exaradas sobre o uso de jogos no contexto da DQ: a maioria dos jogos não segue um gênero específico de jogo, se auto denomina educacional, todos têm origem na academia, são distribuídos gratuitamente, tratam de prevenção do uso de drogas em geral focado em adolescentes e crianças com efetividade normalmente avaliada pelo acréscimo do nível de conhecimento cujo resultado tem sido positivo.

Pela quantidade, constatou-se que há uma carência e oportunidade a ser explorada (o uso de jogos no combate às drogas) mas, ao mesmo tempo, de que este tipo de desenvolvimento (no caso, jogos digitais que promovam algum tipo de mudança de comportamento) não é trivial.

Trabalhos futuros tem, pelo menos, duas direções principais a serem seguidas: (i) explorar o desenvolvimento de exemplos de jogos para poderem ser inseridos no tratamento da dependência química e então, ter-se material para avaliar sua utilidade e efetividade, e; (ii) desenvolver metodologias e abordagens tanto para desenvolvimento desse tipo especial de jogo quanto para a inserção de jogos de um modo geral na rotina da reinserção do indivíduo na sociedade.

\section{Agradecimentos}

Os autores gostariam de agradecer o apoio da FAPESC (Projeto 2013 TR 003705) e da FITEJ (Fundação Instituto Tecnológico de Joinville).

\section{Referências}

ADADE, M.; MONTEIRO, S. 2014 Educação sobre drogas: uma proposta orientada pela redução de danos. Educação e Pesquisa, v. 40, n. 1, p. 215-230.

BOYLE, E., CONNOLLY, T. M., AND HAINEY, T. 2011. The role of psychology in understanding the impact of computer games. Entertainment Computing v. 2, n. 2, p. 69-74.

BUCHINGER, D.; HOUNSELl, M. da S. 2013. Jogos Sérios Competitivo-Colaborativos: Um Mapeamento Sistemático da Literatura. In: Anais do Simpósio Brasileiro de Informática na Educação.

DESHPANDE, A. A., AND HUANG, S. H. 2011. Simulation games in engineering education: A state-of-the-art review. Computer Applications in Engineering Education v. 19, n. 3, p. 399-410.

HAWKINS, J. D., CATALANO, R. F., AND MILLER, J. Y. 1992. Risk and protective factors for alcohol and other drug problems in adolescence and early adulthood: implications for substance abuse prevention. Psychological bulletin v. 112, n. 1, p. 64 .

IMESC, 2014. Instituto de medicina social e de criminologia de São Paulo. Disponível em: http://www.imesc.sp.gov.br/infodrogas/tipoprev.htm. Acesso em Julho de 2014. 
KITCHENHAM, B. et al. 2009. Systematic literature reviews in software engineering-a systematic literature review. Information and software technology, v. 51, n. 1, p. 7-15.

KLISCH, Y., MILLER, L. M., BEIER, M. E., WANG, S. 2012a. Teaching the biological consequences of alcohol abuse through an online game: impacts among secondary students. CBE-Life Sciences Education v. 11, n. 1, p. 94-102

KLISCH, Y., MILLER, L. M., WANG, S., AND EPSTEIN, J. 2012b. The impact of a science education game on students learning and perception of inhalants as body pollutants. Journal of science education and technology 21 , $2,295-303$.

LAMY, F., TERRY B., and PASCAL P.. 2011"SimUse: Simulation of recreational poly-drug use." Artificial Life, 2011 IEEE Symposium on. IEEE.

MARCOS, A; ZAGALO, N. 2011. Instantiating the creation process in digital art for serious games design. Entertainment Computing, v.2, n.2, p. 143-148.

MILLER, L et al. An online, interactive approach to teaching neuroscience to adolescents. CBE-Life Sciences Education, v. 5, n. 2, p. 137-143, 2006.

MONTEIRO, S.; VARGAS, E.; REBELLO, S. Educação, prevenção e drogas: resultados e desdobramentos da avaliação de um jogo educativo: Fundacão Oswaldo Cruz.

PRENSKY, M. 2001. Digital game-based learning: Paragon house. St. Paul, Minnesota, USA.

PETERSEN, K., FELDT, R., MUJTABA, S. MATTSSON, M. 2008. Systematic mapping studies in software engineering. 12th International Conference on Evaluation and Assessment in Software Engineering.

RODRIGUEZ, D M.; TEESSON, M; NEWTON, N C. A systematic review of computerised serious educational games about alcohol and other drugs for adolescents. Drug and alcohol review, v. 33, n. 2, p. 129-135, 2014.

ROMANO, Daniela NARCSim an agent-based illegal drug market simulation;Comput. Sci., Univ. of Sheffield, Sheffield, UK

SONG, H, et al. 2013. "Anti-smoking educational game using avatars as visualized possible selves."Computers in Human Behavior 29.5: 2029-2036.

SZCZESNA A, TOMASZEK M, WIETESKA A. The methodology of designing serious games for children and adolescents focused on psychological goals. In: Pie tka E, KAWA J, EDS. Information technologies in biomedicine. Berlin and Heidelberg: Springer. 2012:245-55.

VERDUIN, M. L. et al. 2013. Computer simulation games as an adjunct for treatment in male veterans with alcohol use disorder. Journal of substance abuse treatment, v. 44, n. 3, p. 316-322.

WILLIAMS, A. V., MEYER, E., AND PECHANSKY, F. 2007. Desenvolvimento de um jogo terapêutico para prevenção da recaída e motivação para mudança em jovens usuários de drogas. Psicologia: Teoria e Pesquisa v. 23, n. 4 , p. $407-414$. 


\begin{tabular}{|c|c|c|c|c|c|c|c|}
\hline Titulo & Super Smoky & Guardian Angel & $\begin{array}{c}\text { The Reconstructors: Nothing to } \\
\text { Rave About }\end{array}$ & $\begin{array}{l}\text { The Reconstructors: } \\
\text { Uncommon Scents }\end{array}$ & N-SquadWeb Adventure & Jogo da Onda & Jogo da Escolha \\
\hline Autor & Song et al. & Verduin et al. & Miller et al. & Klisch et al. & Klisch et al. & Adade e Monteiro & Williams, Anna Virginiz \\
\hline Ano & 2013 & 2013 & 2006 & 2011 & 2011 & 2014 & 2007 \\
\hline IGJ & Aventura & Estratégia & Perguntas e respostas & Influência Social & Perguntas e respostas & Tabuleiro & Cartas/mental \\
\hline $\mathrm{ADN}$ & Educacional & Educacional & Educacional & Educacional & Educacional & Educacional & Jogo sério \\
\hline ORJ & Academia & Academia & Academia & Academia & Academia & Academia & 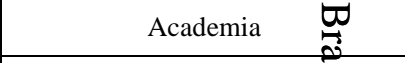 \\
\hline MID & Software & Software & Online & Online & Online & Tabuleiro & Cartas \\
\hline LIC & - & - & Proprietária - Grátis & Proprietária - Grátis & Proprietária - Grátis & - & . \\
\hline DAL & Cigarro & Álcool & Drogas em geral & Inalantes & Álcool & Ilicitas e Lícitas & Drogas em geral $\frac{0}{8}$ \\
\hline PAL & Estudantes do ensino médio. & Maiores de 45 anos & Estudantes do ensino médio & Estudantes do ensino médio & Estudantes do ensino médio & Estudantes do ensino médio & Jovens - Homens e mulh \\
\hline EDQ & Prevenção & Durante tratamento & Prevenção & Prevenção & Prevenção & Prevenção & Recaída \\
\hline MAV & Conhecimento & Conhecimento & Conhecimento & Conhecimento & Conhecimento & Conhecimento & 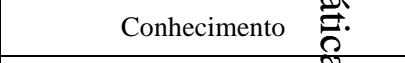 \\
\hline RES & Positivo & Positivo & Positivo & Positivo & Positivo & Positivo & Inconclusivo \\
\hline Descrição & $\begin{array}{l}\text { O jogador controla um } \\
\text { personagem que deve desviar } \\
\text { de inimigos, caracterizados } \\
\text { como zumbis segurando } \\
\text { cigarros Quanto mais } \\
\text { inimigos entrarem em contato } \\
\text { com o avatar, mais a face do } \\
\text { avatar vai envelhecendo. }\end{array}$ & $\begin{array}{l}\text { O jogador deve auxiliar o } \\
\text { protagonista em diversas } \\
\text { atividades do cotidiano, como } \\
\text { retirar da sua casa objetos que } \\
\text { remetem ao alcolismo, e tomar } \\
\text { decisões a respeito do } \\
\text { convívio social. }\end{array}$ & \begin{tabular}{|lr} 
O jogo apresenta histórias \\
ambientadas no futuro que \\
demonstram & problemas \\
relacionados ao uso de drogas em \\
geral. O jogador aprende sobre \\
este problemas e então participa \\
de um jogo de perguntas e \\
respostas para testar o \\
conhecimento adquirido.
\end{tabular} & 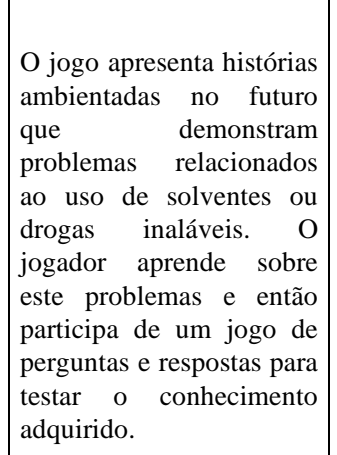 & $\begin{array}{l}\text { O jogo é ambientado no } \\
\text { futuro, e possui animações } \\
\text { que demonstram os problemas } \\
\text { que uma pessoa tem quando } \\
\text { dirige alcoolizada. }\end{array}$ & $\begin{array}{l}\text { Jogo de tabuleiro cujo objetivo } \\
\text { é ser o primeiro jogador a } \\
\text { chegar no centro do tabuleiro. } \\
\text { Para tal, o jogador deve } \\
\text { responder questões que } \\
\text { definidas pelas células } \\
\text { compóem } \\
\text { tabuleiro. }\end{array}$ & 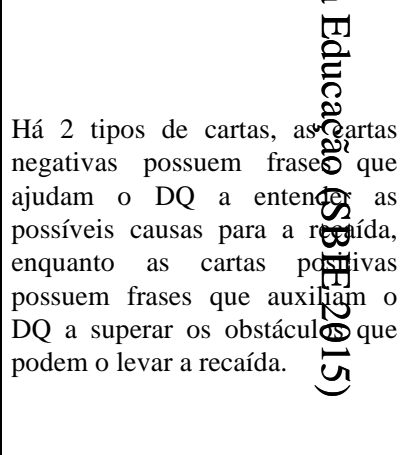 \\
\hline
\end{tabular}

